Document downloaded from:

http://hdl.handle.net/10251/125580

This paper must be cited as:

Barbi, M.; Garcia-Pardo, C.; Nevárez, A.; Pons Beltrán, V.; Cardona Marcet, N. (2019). UWB RSS-based Localization for Capsule Endoscopy using a Multilayer Phantom and In Vivo Measurements. IEEE Transactions on Antennas and Propagation. 67(8):5035-5043. https://doi.org/10.1109/TAP.2019.2916629

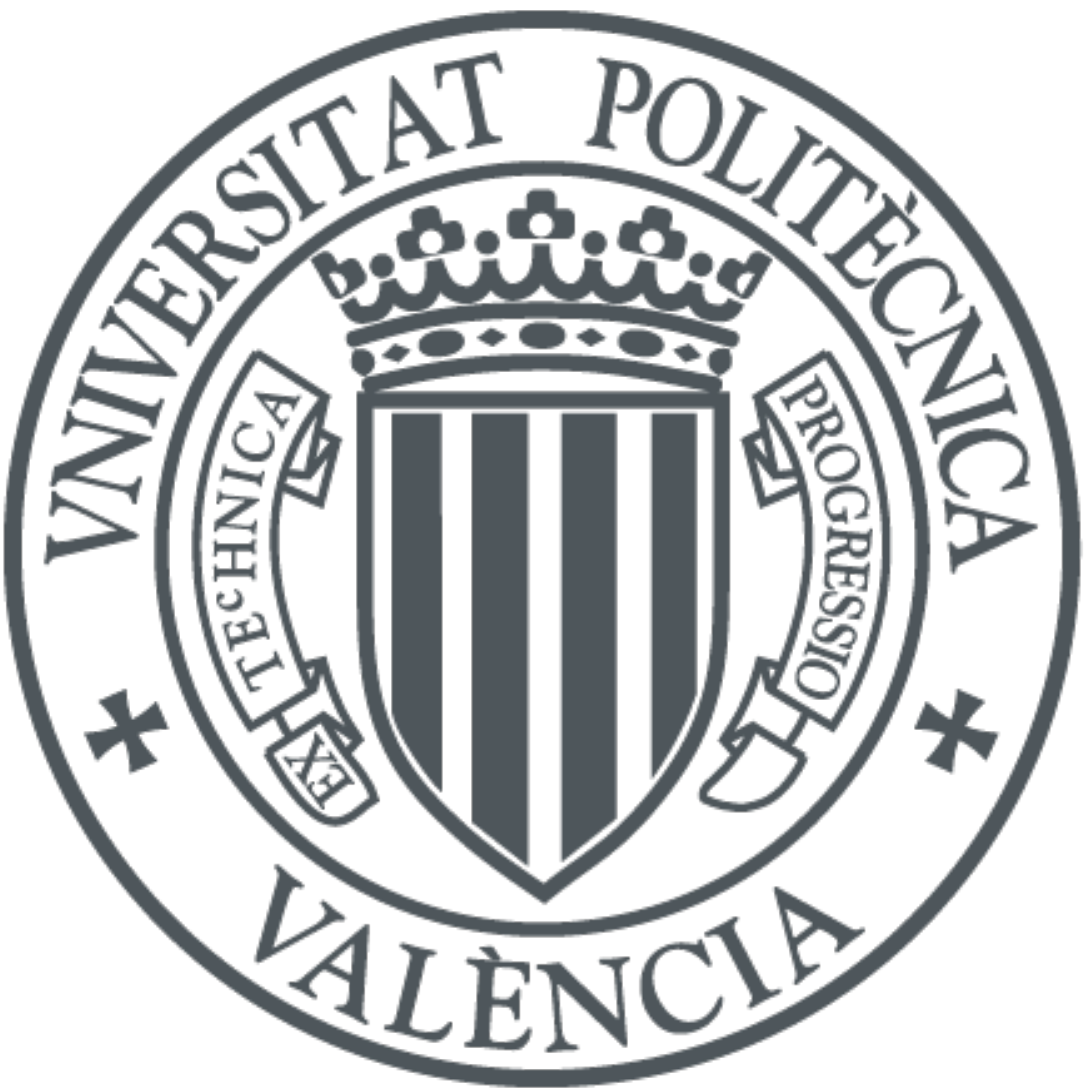

The final publication is available at

https://doi.org/10.1109/TAP.2019.2916629

Copyright Institute of Electrical and Electronics Engineers

Additional Information 


\title{
UWB RSS-based Localization for Capsule Endoscopy using a Multilayer Phantom and In Vivo Measurements
}

\author{
Martina Barbi, Concepcion Garcia-Pardo, Andrea Nevarez, \\ Vicente Pons, Narcis Cardona, Member, IEEE
}

\begin{abstract}
In recent years, localization for capsule endoscopy applications using Ultra-Wideband (UWB) technology has become an attractive field of investigation due to its potential benefits for patients. Literature concerning performance analysis of RF-based localization techniques for in-body applications at UWB frequencies is very limited. Available studies mainly rely on Finite Difference Time Domain (FDTD) simulations, using digital human models and on experimental measurements by means of homogeneous phantoms. Nevertheless, no realistic analysis based on multilayer phantom measurements or through in vivo experiment has been reported yet. This paper investigates the performance of RSS-based approach for two-dimensional (2D) and three-dimensional localization (3D) in the UWB frequency band. For 2D localization, experimental laboratory measurements using a two-layer phantom based setup have been conducted. For 3D localization, data from a recently conducted in vivo experiment have been used. Localization accuracy using path loss (PL) models, under ideal and not ideal channel estimation assumptions, are compared. Results show that under not ideal channel assumption the relative localization error slightly increases for the $2 \mathrm{D}$ case but not for the in vivo $3 \mathrm{D}$ case. Impact of receivers selection on the localization accuracy has also been investigated for both, 2D and 3D, cases.
\end{abstract}

Index Terms-Wireless capsule endoscopy (WCE), UltraWideband (UWB), in-body localization, heterogeneous phantom, in vivo measurements.

\section{INTRODUCTION}

Wireless Capsule Endoscopy (WCE) is a revolutionary invention introduced in the medical sector several years ago. A tiny capsule, equipped with a camera, is swallowed by the patient. As it travels along the gastrointestinal (GI) tract, it takes thousands of pictures (up to 6 per seconds) and sends

This work was supported by the European Union's H2020:MSCA:ITN program for the "Wireless In-body Environment Communication- WiBEC" project under the grant agreement no. 675353. This work was also funded by the Programa de Ayudas de Investigación y Desarrollo (PAID-01-16) from Universitat Politècnica de València and by the Ministerio de Economía y Competitividad, Spain (TEC2014-60258-C2-1-R), by the European FEDER funds.

M. Barbi, C. Garcia-Pardo, N. Cardona are with iTEAM, Universitat Politècnica de València, 46022 Valencia, Spain (e-mail: \{marbar6, cgpardo, ncardona\}@iteam.upv.es).

A. Nevarez and V. Pons are with Hospital Universitari i Politècnic La Fe, Digestive Diseases Area, 46126 Valencia (email: nevarezja78@gmail.com, pons_vicbel@gva.es). them to an external recorder located on a belt around the patient's waist. When the recorder is returned, the physician is able to visualize a video of the whole patient's small bowel and detect potential abnormalities by visual inspection. However, the images provided by current capsule endoscope are not very high quality [1]. Moreover, a precise localization of anomalies is highly important for their subsequent treatment. In fact, the position estimation of the pill is currently very inaccurate by using proprietary software applications provided to hospitals [2], [3] so the doctor cannot precisely locate detected diseases. Besides, not only precise location but also an accurate tracking of the capsule is of fundamental importance for the future of this technology [4].

In literature, many different approaches are available to locate the capsule endoscope. Several techniques use the radio frequency (RF) signals [5], [6], others magnetic fields [7], [8] and others imaging processing techniques [9], [10], [11].

RF-based localization inside the human body is an evolution of RF technology applied to indoor localization. Therefore, in literature, same techniques used for indoor localization are being investigated and adapted for capsule endoscope localization. However, in this case, the presence of the body tissues, instead of air, is even more problematic for the localization procedure, specifically in the Ultra-Wideband (UWB) frequency band. In fact, the human body consists of different types of tissue each having its own electromagnetic properties. Particularly, permittivity and conductivity vary over the different tissues and present values much higher than those of the air. Moreover, these electromagnetic properties are frequency-dependent. As a consequence, the RF signal at UWB frequencies suffers from large frequency-dependent attenuation and severe multipath conditions which makes ranging distance estimation very challenging. Despite these issues the use of the RF signal, that the WCE uses for images transmission, also for localization purposes constitutes an optimal solution to keep the hardware of the capsule simple. Through this approach, firstly, a ranging estimation is performed. Secondly, the coordinates of the in-body source are calculated through trilateration methods. Ranging estimation is commonly accomplished by using distancedependent parameters such as received signal strength (RSS) [12], time of arrival (ToA) [12], [13], time difference of arrival (TDoA) [13] or phase difference of arrival (PDoA) 
[14], [15]. In particular, RSS-based localization, is commonly implemented due to its simplicity [12], [16]. Using this approach, the ranging estimation is performed estimating the attenuation suffered by the received signal and relating this with a previously established model of the attenuation as a function of the distance from the source (path loss model). Thus, the localization performance highly relies on the accuracy of the model [4].

Current capsule endoscopes operate in the MICS frequency band (402-405 MHz). Although this band offers good penetration of the signal for in-body applications, the data rate (up to $500 \mathrm{Kbps}$ ) is too low to support high quality images [1]. In order to achieve this goal, in recent years Ultra-Wideband (UWB) technology, has been under investigation for future capsule endoscopes due to its many advantages [17]. Particularly, the lowest part of the UWB spectrum (3.1-5.1 $\mathrm{GHz}$ ) is being considered in literature, due to the unaffordable signal attenuation above this frequency range [18].

Currently, performance analysis of RF-based localization techniques in the MICS frequency band are widely available [19]. On the contrary, studies conducted in the UWB frequency band are very limited. For RSS-based ranging, the main issue is the lack of standardized path loss models for inbody to on-body communications at UWB frequencies.

Results through RF-based signals and compressive sensing are obtained in [16] using Computer Simulation Technology (CST) simulator with Finite Integration Technique (FIT) solver in 1-3 and 3-5 GHz frequency bands. Best performance showed a mean localization error of $40 \mathrm{~mm}$. RSS-based approach is investigated in [20], through FIT simulator, in 1-6 $\mathrm{GHz}$ frequency band, showing a localization accuracy in the $\mathrm{cm}$ range. Authors in [21], address the crucial problem of ranging with UWB signals inside the human body using XFDTD software platform, in 3.4-4.8 GHz frequency range. Measurements campaign at $2-2.4 \mathrm{GHz}$ using a homogeneous phantom model to investigate the influence of body tissue on the accuracy of ToA-based ranging technique is presented in [22]. Besides possible inaccuracy of the UWB phantom model used, homogeneous phantoms only emulate one human tissue therefore they cannot model with sufficient accuracy the complex human body scenario.

Performing UWB heterogeneous phantoms-based measurements is not as simple as for the homogeneous phantom case because of the complexity to accurately mimic the electromagnetic properties of different human tissues in the whole UWB frequency band. Recently, researchers at Universitat Politècnica de València (UPV) [23], [24], [25] have accomplished with this necessity. Furthermore, a customized measurement setup, presented in Section III.A, has been proposed in order to improve the measurements accuracy for in-body scenarios [26]. Regarding in vivo measurements, experiments in living animals are not easy to conduct as they are subject to ethical restrictions and extremely costly as dedicated facilities and a specialized medical team are required. Despite these difficulties, in vivo measurements, as previously pointed out, is the most realistic approach for inbody radio channel characterization and thus, for the testing of
RF-based localization techniques.

To the best of the author's knowledge no implementation of RF-based localization techniques at UWB frequencies, using either an experimental heterogeneous phantom or through in vivo experiments has been reported yet. Particularly, in vivo measurements are of high relevance in order to test developed localization algorithms in a scenario which is the closest to reality, compared to laboratory measurements (controlled environment) and simulations (ideal environment).

In this paper, the performance of RSS-based localization technique for UWB in-body to on-body (IB2OB) communications are investigated. To this aim, laboratory measurements using a customized multilayer phantom-based testbed as well as in vivo experiment have been conducted. Localization results obtained for both measurement campaigns are then compared, in order to analyze the performance in case of an emulated WCE scenario (laboratory) and in case of a most realistic one (in vivo). Two-dimensional (2D) localization is performed based on the experimental laboratory measurements while three-dimensional (3D) positioning is performed through the in vivo data.

The remainder of the paper is organized as follows: Section II briefly describe the real WCE scenario and the one emulated through experimental measurements. Section III presents the experimental measurements campaign conducted in laboratory as well as the in vivo experiment. Section IV describes the RSS-based ranging technique, assuming ideal and not ideal channel estimation, as well as the localization algorithms used to evaluate the results for the 2D and 3D case. Results are presented and discussed in Section V and Section VI, respectively, along with the future research plans. Finally, Section VII concludes the paper.

\section{APPLICATION SCENARIO}

In current WCE procedures, in order to locate the capsule endoscope, a sensors array [3], as shown in Fig. 1(a), is placed on the patient's body to receive transmission data from the pill while it is moving along the Gastrointestinal (GI) tract.

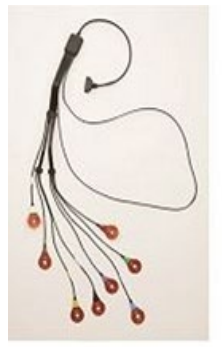

(a)



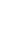

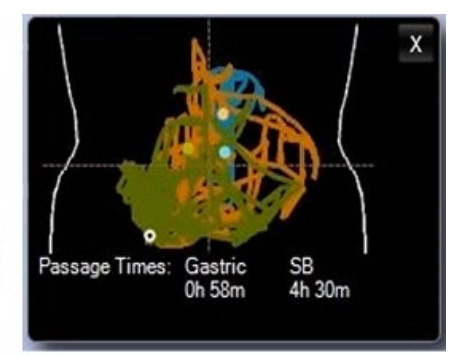

(b)
Fig. 1. Sensor array (a), movement pattern drawn by the software provided by Given Imaging (b) [3]

The information collected by the sensors is then processed offline by the software provided to the hospitals in order to visually draw the movement pattern (Fig. 1(b)) of the capsule travelling along the GI tract, depending on the landmark chosen by the physician. The mean localization error, 
provided by, for example, the widely used Given Imaging software, is roughly $3.8 \mathrm{~cm}[27]$.

With the aim of reproducing the real in-body to on-body scenario (Fig. 1(a)) for localization purposes, laboratory and in vivo measurements, described in Section III.A and III.B, were performed using, as in-body source, a small UWB patch antenna, designed to operate inside the human body [28], since there are currently no capsule endoscopes operating at these frequencies. A receiving UWB patch antenna, specifically designed to operate on the surface of the human body [29] was placed in different on-body locations in order to emulate the sensors array (Fig. 1(a)) currently used in capsule endoscopy procedure. The collected data were then processed off-line in order to locate in 2D and 3D the in-body source, as it will be explained in Section IV.

\section{Measurement SetuP AND Methodology}

\section{A. Phantom measurements}

Experimental measurements, using a multilayer phantombased setup were conducted in the 3.1-8.5 GHz UWB frequency band. A brief description of the testbed (Fig. 2) is given here and further details can be found in [26].

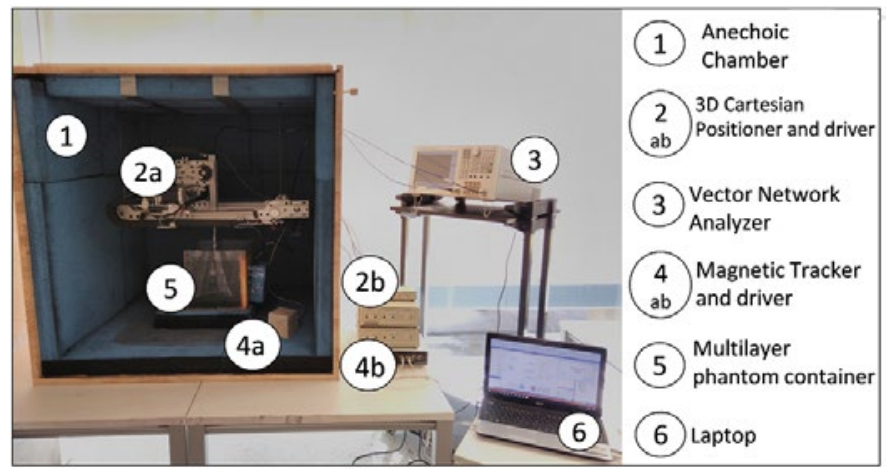

Fig. 2. Measurement Testbed [26]

All the equipment involved in the setup is software-driven by a laptop (Fig. 2, element 6). The 3D Cartesian positioner (Fig. 2, element 2a) accurately moves the in-body antenna along XYZ axis inside the two-layer phantom container (Fig. 2, element 5).

The WCE scenario involves different human tissues, mainly colon, muscle and fat. As colon and muscle have similar permittivity, the phantom container was designed specifically for muscle and fat layers. Muscle phantom is widely used in literature for in-body to on-body communications studies. Moreover, the one created at UPV [24] precisely covers the whole UWB frequency band and it is the most accurate so far in literature.

A magnetic sensor is attached to the in-body and on-body antenna so that the tracker (Fig. 2, element 4ab) can precisely evaluate the distance between antennas as well as their respective coordinates according to the magnetic transmitter reference system (Fig. 3).

Measurements were performed by moving the in-body antenna, placed inside the muscle layer, in steps of $1 \mathrm{~cm}$ along $\mathrm{x}, \mathrm{y}, \mathrm{z}$ axis with grid size of $(\mathrm{Nx}=12, \mathrm{Ny}=11, \mathrm{Nz}=2)$, as depicted in Fig. 3. Five on-body antenna positions, with a separation of $2 \mathrm{~cm}$, along $\mathrm{y}$ as well as along $\mathrm{z}$ axis, were considered on the outer edge of the fat layer (Fig. 3).

In order to improve the Signal-to-Noise Ratio (SNR) for each in-body antenna position on the grid and each on-body receiver, five snapshots of the channel $\left(S_{21}\right)$ were taken, considering 3201 resolution points in frequency. Only values above the noise level $(-90 \mathrm{~dB})$ were taken into account. For each snapshot the tracker calculated the distance between antennas, as well as in-body and on-body $(\mathrm{x}, \mathrm{y}, \mathrm{z})$ antenna coordinates, 100 times.

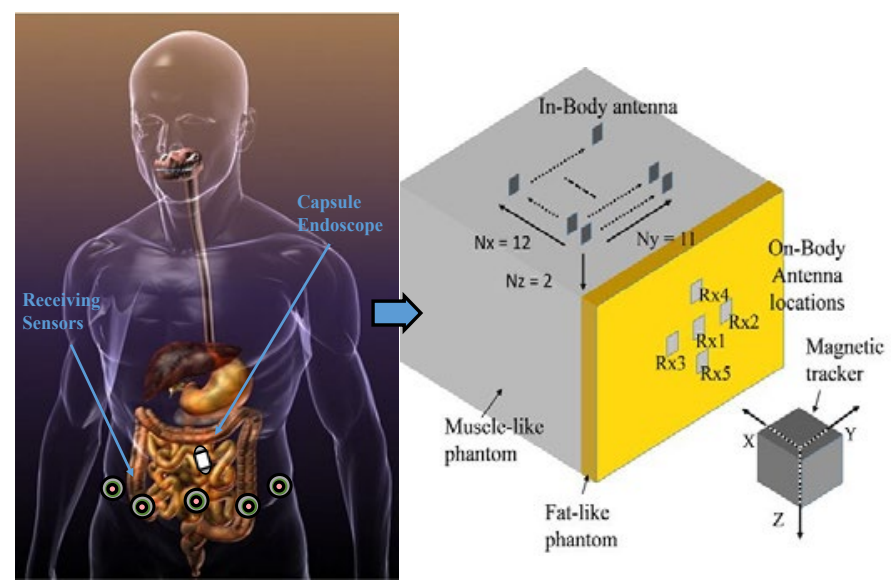

Fig. 3. WCE procedure vs Experimental Measured Grid

By averaging the collected data, $S_{21}$, antenna separation and antennas coordinates were evaluated.

\section{B. In vivo measurements}

In vivo measurements were conducted in a living porcine model, at the animal laboratory of the Hospital Universitari i Politècnic la $\mathrm{Fe}$ in Valencia, Spain, after approval by the Ethical Committee of Investigation of the hospital, under the protocol WIBEC 2015/0463. A brief description of the experiment is given in this section, in order to highlight the main aspects. Further and more detailed informations can be found in [30].

Same equipment (VNA and magnetic tracker) described in Section III.A for the phantom measurements campaign was used. As the main scenario of interest for WCE applications is the GI tract, the in-body antenna was placed, through laparoscopy, in three different positions inside the abdomen of the porcine model, in order to be surrounded by either small bowel or colon or both. For each in-body location, the onbody antenna was placed on the abdomen of a porcine model, in direct contact with the skin (Fig. 4(a)), in thirteen different locations, as depicted in Fig. 4(b).

Measurements were taken in the 3-6 GHz UWB frequency band, considering 1601 resolution points in frequency. Through the VNA, for each pair of in-body to on-body positions five snapshots of the channel $\left(\mathrm{S}_{21}\right)$ were taken. Again, only values above the noise level $(-90 \mathrm{~dB})$ were taken into account.

As for the phantom measurements, antenna separation distance and antenna coordinates were evaluated 100 times per snapshot by the magnetic tracker. Finally, averaging the 
collected measurements, $\mathrm{S}_{21}$, antennas separation distance and antennas coordinates were evaluated.

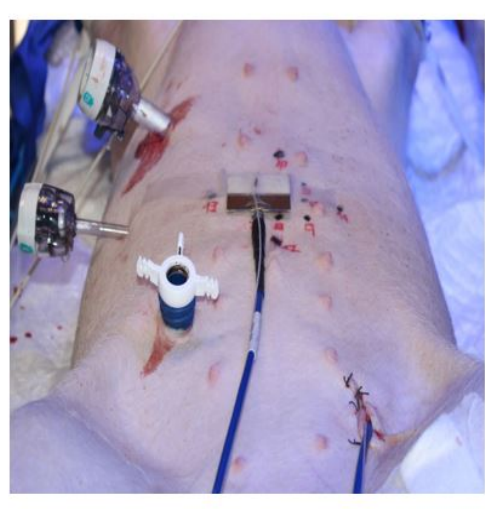

(a)

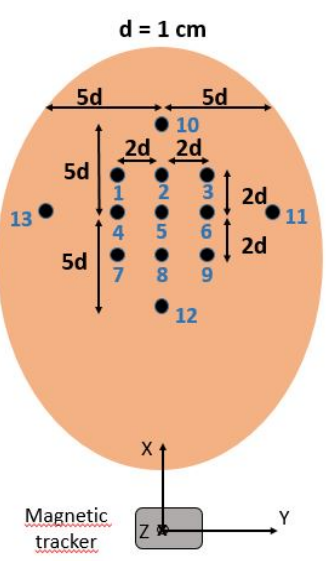

(b)
Fig. 4. In vivo measurements for one on-body position (a), and measured grid for each in-body position (top view) (b)

\section{RSS-BASED RANGING \& LOCALIZATION ALGORITHMS}

For both measurement campaigns, data from $3.1-5.1 \mathrm{GHz}$ are considered because for antenna distances larger than 8-9 $\mathrm{cm}$ measurements beyond $5.1 \mathrm{GHz}$ are below the noise level [26].

From the measured $S_{2 l}$, the path loss is evaluated for each in-body to on-body antenna position as follows:

$$
P L_{\text {meas }}(d B)=-10 \log _{10}\left(\operatorname{mean}\left(|H(f)|^{2}\right)\right)
$$

where $H(f)$ is the frequency-domain transfer function in $N$ resolution points computed as $H(f)=\left|S_{21}\right| e^{-j \phi s_{21}}$, being $\left|S_{21}\right|$ and $\phi_{S_{21}}$ module and phase in radians of the $S_{21}$, respectively.

For both measurement campaigns, path loss values within a distance of $8 \mathrm{~cm}$ between in-body and on-body antenna centers are selected due to the noise level, as pointed out at the beginning of the section.

Selected path loss values are then fitted by a log-distance approximation model:

$$
P L(d B)=P L_{0, d_{r e f}}(d B)+10 n \log _{10}\left(\frac{d}{d_{r e f}}\right)
$$

where $d$ is the distance between antenna centers, $d_{r e f}$ is the reference distance at $1 \mathrm{~cm}, P L_{0, d_{r e f}}$ is the path loss at $d_{r e f}$ and $n$ is the path loss exponent.

From measurements, the performance of RSS-based positioning is evaluated and compared under two different assumptions:

1. Assuming an ideal receiver capable of detecting all the multipath components of the channel impulse response (ideal case) so through (1) the path loss can be precisely estimated as:

$$
P L_{e s t}(d B)=P L_{\text {meas }}(d B)
$$

2. Considering a real case scenario, where the receiver receives for a given period of time being able to detect only few multipath components with power below a certain level from the strongest path. In this case, the path loss can be computed as follows:

$$
P L_{e s t}(d B)=-10 \log _{10} \operatorname{sum}\left(\left.h(\tau)\right|_{\text {sel }} ^{2}\right)
$$

where $|h(\tau)|_{\text {sel }}=|\operatorname{ifft}(H(f))|_{\text {sel }}$ are the selected multipath components of the channel impulse response.

From the model proposed in (2) an estimation of $d$ can be obtained as follows:

$$
d_{e s t}=10 \frac{P L_{e s t}-P L_{0, d_{r e f}}}{10 n} \cdot d_{\text {ref }}
$$

where $P L_{\text {est }}$ is the path loss evaluated using (3) or (4) if assuming ideal or not ideal channel estimation, respectively.

\section{A. 2D Phantom-Based Post-processing}

For the multilayer phantom measurements only two coordinates of the in-body antenna ( $\mathrm{y}$ and $\mathrm{z}$ ) can be evaluated as all receivers share the same $\mathrm{x}$-coordinate (Fig. 3). In order to estimate the in-body antenna coordinates the adaptive linearized method described in [31] is adapted [32] and implemented for the two dimensional case. Three receivers are needed to find the unique solution of the linearized system of two equations in two unknowns. No more than three receivers are used to evaluate the $2 \mathrm{D}$ localization performance. Due to the number of receivers (only five) and their configuration (Fig. 3), using 4 or all 5 receivers and applying minimization error techniques does not improve the estimation accuracy of the in-body antenna coordinates.

\section{B. $3 D$ In vivo Post-processing}

Regarding the in vivo measurements, one of the in-body positions was discarded because the antenna separation distances were above $8 \mathrm{~cm}$ for all on-body receiver positions.

A general path loss model is calculated through (2) considering all the on-body antenna locations (Fig. 4(b)) and the two in-body positions under study. For 3D localization, if applying the adaptive linearized method, four receivers are needed to directly solve the system of three equations in three unknowns. Since in vivo measurements are not as many as those performed in laboratory with phantom and due to the animal's respiration, path loss values present high standard deviation with respect to the evaluated fitted model [30]. Therefore, for 3D localization the method described in [33] is used. More than four receivers are selected for positioning. A first estimation of the in-body antenna coordinates is obtained through the Linear Least Square method. Then, the NonLinear Least Square (NLLS) approach is applied. The sum of the square errors is minimized through the LevenbergMarquardt algorithm [34]. Further details regarding the selection criteria of the receivers as well as the performance metrics used to evaluate the results are given in Section V. 


\section{RESULTS}

\section{A. Performance Metrics}

In order to assess the achieved localization accuracy, the localization error, $L E$, for the $3 \mathrm{D}$ case and its relative error can be defined as:

$$
\begin{gathered}
L E=\sqrt{\left(x_{I B}-x_{I B_{-} e s t}\right)^{2}+\left(y_{I B}-y_{I B_{-} e s t}\right)^{2}+\left(z_{I B}-z_{I B_{-} e s t}\right)^{2}} \\
\operatorname{Rel} L E=\frac{L E}{\sqrt{x_{I B}^{2}+y_{I B}^{2}+z_{I B}^{2}}}
\end{gathered}
$$

where $\left(x_{I B}, y_{I B}, z_{I B}\right)$ and $\left(x_{I B_{-} e s t}, y_{I B_{-} e s t}, z_{I B_{-} e s t}\right)$ are the real and estimated coordinates of the in-body antenna, respectively.

For the 2D case, (6) and (7) are calculated omitting the xcoordinate as for the specific receivers configuration (Fig. 3) it cannot be estimated.

Moreover, the relative errors on the estimation of the inbody antenna coordinates can be evaluated individually as follows:

$$
\begin{aligned}
& \operatorname{RelErr}_{x_{I B}}=\frac{\left|x_{I B \_e s t}-x_{I B}\right|}{\left|x_{I B}\right|} \\
& \operatorname{RelErr}_{y_{I B}}=\frac{\left|y_{I B \_e s t}-y_{I B}\right|}{\left|y_{I B}\right|} \\
& \operatorname{RelErr}_{z_{I B}}=\frac{\mid z_{I B \_} e s t}{\left|z_{I B}\right|}-z_{I B} \mid
\end{aligned}
$$

\section{B. 2D Localization Results}

As detailed in Section IV, for the multilayer phantom-based measurements path loss values related to antennas distances up to $8 \mathrm{~cm}$ are fitted through (2), being $d_{0}=1 \mathrm{~cm}, P L_{0, d r e f}=-$ $24.43 \mathrm{~dB}$ and $n=9.69$.

In order to estimate the $\left(y_{I B}, z_{I B}\right)$ coordinates of the in-body antenna different combination of three receivers (one taken as reference) were considered to directly solve the linearized system in [31] with two equations. Fig. 5(a) depicts the Cumulative Distribution Function (CDF) of the relative localization error, computed as in (7), for three different combinations of three receivers. In Fig. 5(b) the true locations of the in-body antenna (given by the magnetic tracker) are represented versus the estimated ones for the same combinations of receivers. Results show how the receivers selected for localization impact the accuracy of the results. Particularly, the combination of receivers 2,4 and 3, taken as reference, leads to lower relative error values compared to the other combinations, as it is experiencing on average the highest level of received power [35], [36]. Similar results were obtained for the same combinations of receivers in a narrower frequency band (3.1-4.1 GHz) [32].
Considering the combination of receivers leading to the best performance, i.e. receivers 2, 4 and 3 as reference (Fig. 5), localization accuracy was evaluated and compared in case of ideal and not ideal channel estimation assumption, detailed in Section IV. As already mentioned, in case of ideal channel (case 1), the path loss is computed as in (1). In case of not ideal channel (case 2) the path loss is evaluated through (4), by selecting all the multipath components whose power is above or equal to the maximum of the Power Delay Profile minus a certain threshold, specifically $5 \mathrm{~dB}, 10 \mathrm{~dB}, 20 \mathrm{~dB}$, respectively.
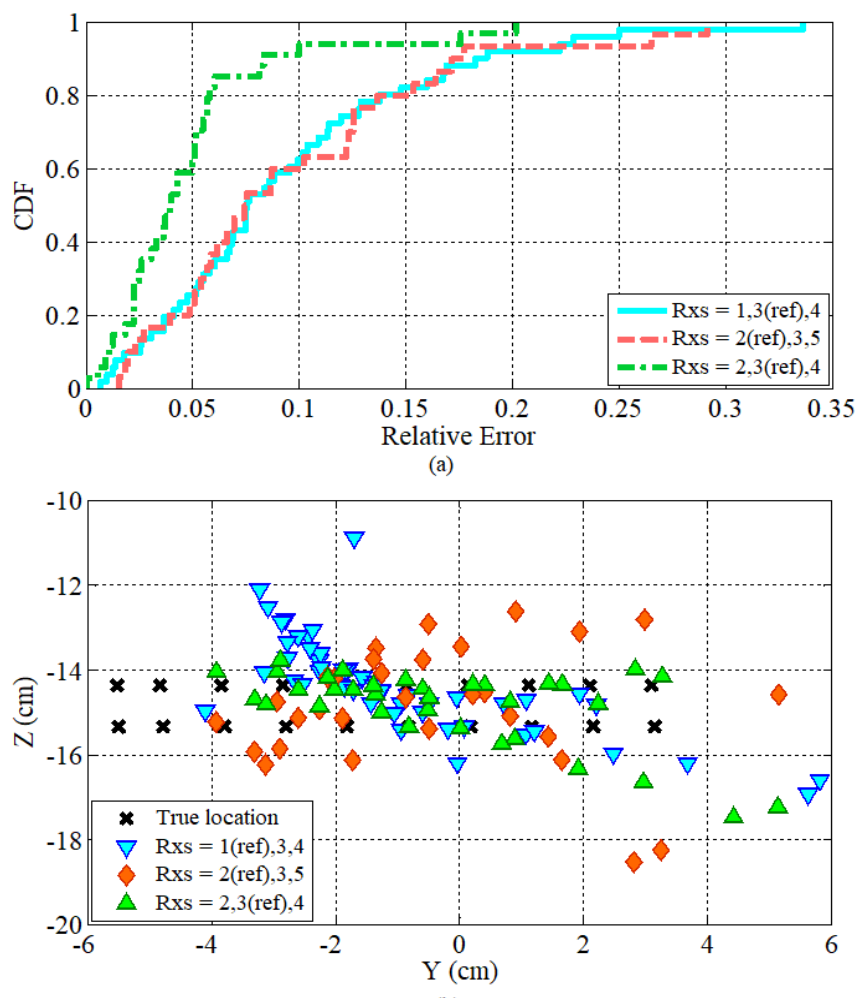

Fig. 5. CDF of relative localization error (a), and true vs estimated location of in-body antenna (b)

Fig. 6(a) shows that considering the components with power below 10/20 $\mathrm{dB}$ from the maximum (magenta and green curve), almost same performance as for the ideal channel case are obtained, while slightly worse performance is observed when considering the components below $5 \mathrm{~dB}$ (blue curve). This is also noticeable in Fig. 6(b) where, using the same combination of receivers, an example of tracking considering the in-body antenna moving in steps of $1 \mathrm{~cm}$ along $y$-axis, for $x=1$ and $z=2$, is presented. It is important to mention that for this track, the distance between the in-body antenna and some on-body receivers is sometimes higher than $8 \mathrm{~cm}$. Thus, for such points the path loss model used for ranging estimation does not include such distances, leading to some inaccuracies. In fact, it is worth observing in Fig. 6(b) that for all the considered cases, the estimation error is higher for the outer points of movement of the in-body antenna i.e., $1-4,10,11$. For these positions the distance between in-body antenna and receivers 2, 3 is outside the region of validity of the evaluated path loss model. This means more inaccuracy in the ranging distance calculation in (5) and consequently more 
uncertainty in the estimation of the in-body antenna coordinates $\left(y_{I B}, z_{I B}\right)$.

Regarding the localization performance, considering ideal channel estimation (case 1) an average relative localization error of $4.7 \%$ corresponding to a $\mathrm{LE}=0.72 \mathrm{~cm}$ is achieved. For not ideal channel estimation (case 2), considering components with power below $5 \mathrm{~dB}$ from the maximum, an average relative error of $5.7 \%(\mathrm{LE}=0.86 \mathrm{~cm})$ is obtained. This means that in a realistic scenario (not ideal channel estimation) the inability of the receiver to perfectly characterize the channel affects the positioning accuracy leading to an increase of $1 \%$ in the localization error, in this case under study.

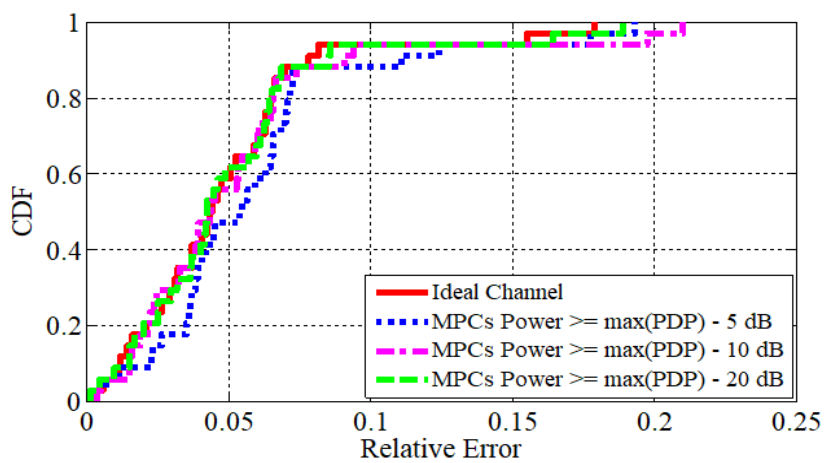

(a)



(b)

Fig. 6. Relative localization error (a) and example of tracking for in-body antenna moving along $y$-axis, for $x=1$ and $z=2$ (b), for ideal and not ideal channel

\section{3D Localization Results}

For the in vivo measurements, as detailed in Section IV, path loss values related to antennas distances below or equal to $8 \mathrm{~cm}$ are fitted through (2), as for the $2 \mathrm{D}$ case, resulting $d_{0}=1 \mathrm{~cm}, P L_{0, d r e f}=21.84 \mathrm{~dB}$ and $n=5.44$. In this case the dispersion of the path loss values with respect to the fitting model is higher (RMSE 28) [30]. This is due to the fact that measurements were conducted in a realistic and different scenario with respect to the laboratory environment. Moreover, much less measurement points with respect to the laboratory measurements campaign, are available to derived a path loss model, as mentioned in Section IV.B.

In order to compare the goodness of the localization method, in-body antenna coordinates were firstly estimated as for the $2 \mathrm{D}$ case, i.e. by solving the linearized system in [31] using four receivers (3D case). Performance in terms of relative localization error, computed as in (7), are presented in Table I. Labels Tx1 and Tx2 indicate the error values related to the first and the second in-body position under study, respectively. Results show that this localization method, unlike for the 2D case, is not suitable in this realistic scenario, leading to high inaccuracy, especially for in-body position Tx2. In order to improve the performance, as mentioned in Section IV.B, the NLLS method was applied to minimize possible inaccuracy in the ranging distance estimation through the derived path loss model. Results obtained by applying such method for different number of receivers are presented in Table I. For each case, receivers experiencing the highest relative received power were selected.

Results in Table I show that implementing the NLLS method significantly improves the estimation accuracy, especially for the second in-body position whose relative error drops up to less than $1.5 \%$, by using $7 / 10$ receivers. However, by increasing the number of receivers from 7 to 10 and from 7 to 13 the relative localization error slightly decreases for inbody position Tx1 but not for position Tx2. In fact, passing from 10 to 13 receivers means, for in-body position $T x 1$ having one receiver outside the region of validity of the path loss model $(\geq 8 \mathrm{~cm})$ and for position Tx2 having three of them. This clearly affects the ranging accuracy and adds uncertainty when applying the minimization error algorithm. As for both in-body positions increasing the number of receivers up to 10 leads to fairly good performance, results presented in the remainder of this section were obtained applying the Levenberg-Marquardt algorithm by selecting those ten receivers experiencing the highest power.

TABLE I

LOCALIZATION ERRORS FOR DIFFERENT APPROACHES

\begin{tabular}{lll}
\hline \hline & \multicolumn{2}{c}{$\operatorname{Rel}_{L E}(\%)$} \\
\multicolumn{1}{c}{ Method } & $\mathrm{Tx} 1$ & $\mathrm{Tx} 2$ \\
\hline \hline Linearized System & 38.5 & 2848 \\
NLLS (7 Rxs) & 1.81 & 1.41 \\
NLLS (10 Rxs) & 1.48 & 1.40 \\
NLLS (13 Rxs) & 1.21 & 2.82 \\
\hline \hline
\end{tabular}

In order to compare the goodness of the obtained path loss model for the 2D case, 3D localization performance were evaluated by using the phantom path loss model given in Section V.B. Table II compares the relative localization error obtained using the general path loss model obtained from the in vivo measurements with the one derived from the multilayer phantom-based measurements. Using the phantom model leads to an increment of 3-4 \% in the localization error.

TABLE II

LOCALIZATION ERRORS USING DIFFERENT PL MODELS

\begin{tabular}{ccc}
\hline \hline & \multicolumn{2}{c}{$\operatorname{Rel}_{L E}(\%)$} \\
PL Model & Tx1 & Tx2 \\
\hline \hline General In Vivo & 1.52 & 1.66 \\
Phantom-based & 4.48 & 6.13 \\
\hline \hline
\end{tabular}

As explained at the beginning of this section, due to the differences between the two path loss models, better results, as 
expected, were obtained by using the fitting model related to the specific scenario (in vivo).

As for the 2D case, performance considering ideal and not ideal channel were evaluated. Table III and Table IV report, for in-body position $\mathrm{Tx} 1$ and $\mathrm{Tx} 2$, respectively, the localization error $L E$ and its relative error $\operatorname{Rel}_{L E}$ as well as the relative and absolute errors on the estimation of $x_{I B}, y_{I B}$ and $z_{I B}$ for ideal and not ideal channel case, using the general in vivo path loss model.

TABLE III

LOCALIZATION ERRORS FOR IN-BODY POSITION TX1

\begin{tabular}{lccc}
\hline \hline & Ideal & $-10 \mathrm{~dB}$ & $-5 \mathrm{~dB}$ \\
\hline \hline $\operatorname{Rel}_{L E}(\%)$ & 1.48 & 1.27 & 1.11 \\
$\operatorname{LE}(\mathrm{cm})$ & 0.97 & 0.83 & 0.72 \\
\hline $\operatorname{RelErr}_{x_{I B}}(\%)$ & 1.03 & 0.93 & 0.81 \\
$\operatorname{AbsErr}_{x_{I B}}(\mathrm{~cm})$ & 0.66 & 0.60 & 0.52 \\
\hline $\operatorname{RelErr}_{y_{I B}}(\%)$ & 5.93 & 4.85 & 2.87 \\
$\operatorname{AbsErr}_{y_{I B}}(\mathrm{~cm})$ & 0.28 & 0.23 & 0.14 \\
\hline $\operatorname{RelErr}_{Z_{I B}}(\%)$ & 6.40 & 5.19 & 4.77 \\
$\operatorname{AbsErr}_{z_{I B}}(\mathrm{~cm})$ & 0.65 & 0.52 & 0.48 \\
\hline \hline
\end{tabular}

TABLE IV

LOCALIZATION ERRORS FOR IN-BODY POSITION TX2

\begin{tabular}{lccc}
\hline \hline & Ideal & $-10 \mathrm{~dB}$ & $-5 \mathrm{~dB}$ \\
\hline \hline $\operatorname{Rel}_{L E}(\%)$ & 1.40 & 1.71 & 1.86 \\
$\operatorname{LE}(\mathrm{cm})$ & 0.91 & 1.12 & 1.21 \\
\hline $\operatorname{RelErr}_{x_{I B}}(\%)$ & 0.55 & 0.78 & 0.41 \\
$\operatorname{AbsErr}_{I_{I B}}(\mathrm{~cm})$ & 0.35 & 0.50 & 0.26 \\
\hline $\operatorname{RelErr}_{y_{I B}}(\%)$ & 6.44 & 8.65 & 5.53 \\
$\operatorname{AbsErr}_{y_{I B}}(\mathrm{~cm})$ & 0.21 & 0.29 & 0.18 \\
\hline $\operatorname{RelErr}_{z_{I B}}(\%)$ & 8.34 & 9.81 & 11.99 \\
$\operatorname{AbsErr}_{I_{I B}}(\mathrm{~cm})$ & 0.82 & 0.96 & 1.17 \\
\hline \hline
\end{tabular}

Results, considering ideal and not ideal channel, show that the lowest localization errors are obtained in the estimation of $x_{I B}$ for both in-body positions. As reported in Table III, for inbody position $\mathrm{Tx} 1$ error values calculated considering ideal channel are slightly higher than those obtained for the not ideal channel case. For in-body position Tx2 same behavior is observed in the estimation of $x_{I B}$ and $y_{I B}$, in Table IV. Although the difference between error values in both cases is not critical, this closely depends on the evaluated path loss model, in Section IV.B. As pointed out in the same section, few in vivo measurement points are available to derive a model. In fact, as detailed in [30], measured path loss values present a higher standard deviation with respect to the calculated fitting model, compared to the 2D case. Moreover, the pig's respiration might also have been affecting the relative received power and thus the accuracy of the localization.

\section{DISCUSSION \& FUTURE WORK}

Summarizing the results, it was found that for the 2D case the combination of three receivers experiencing the highest power leads to the best performance in terms of relative localization error. This is in line with the way current WCE localization algorithms work [36], [37]. Considering ideal channel estimation an average relative localization error of $4.7 \%(0.72 \mathrm{~cm})$ has been obtained. For not ideal channel estimation case, the accuracy slightly decreases, as expected, resulting into a relative localization error on average of $5.7 \%$ $(0.86 \mathrm{~cm})$.

For 3D localization, best performance was achieved by selecting those ten receivers experiencing the highest received power. In case of ideal channel estimation, considering both in-body locations under study, an average relative localization error of $1.4 \%(0.94 \mathrm{~cm})$ has been obtained. For not ideal channel estimation case, the localization error is slightly lower, with respect to the ideal case, due to the derived path loss model.

It is important to mention that for both, laboratory and in vivo measurements, the orientation of the in-body and on-body antenna was kept the same, in order to better investigate the effect of the propagation channel on the localization performance. In real applications, this condition is not satisfied at all and the unknown orientation of the capsule inside the GI tract affects the localization accuracy. As part of the future work, further experimental measurements could be performed to take into account the misorientation between antennas. As a matter of fact, the directionality (or null) in the radiation pattern of the transmitting antenna could be exploited to estimate its orientation through several on-body receivers, as presented in a recent study in [38].

Additionally, a possible solution to overcome signal losses, due to orientation changes of the in-body antenna, could be the use of circularly polarized in-body antennas which are less vulnerable to polarization mismatches and multipath distortion.

Results presented in this paper show the importance of the path loss model for localization using the received signal strength. Although the methodology used is valid for any separating distance between in-body and on-body antenna, the derived path loss models are not valid for antennas distance above $8 \mathrm{~cm}$ due to our particular measurement setup. More extensive measurements campaigns are being arranged in order to derive a more accurate and general path loss model as well as, in case of in vivo experiment, further studies on the impact of the animal's respiration on the received power are being conducted.

Finally, combination of RSS-based approach with other localization technique (image-based, for example) will be explored in order to improve the positioning accuracy.

\section{CONCLUSIONS}

In this paper, performance of RSS-based localization technique, for in-body to on-body communications in the 3.15-1 GHz UWB frequency band have been investigated through experimental laboratory measurements and in vivo experiment. 2D localization is performed using experimental measurements conducted through a customized multilayer 
phantom-based testbed. In this case, an adaptive linearized method, considering different combinations of three receivers, is sufficient to estimate the in-body antenna coordinates. 3D localization is performed using data collected during a recently conducted in vivo experiment in a living pig. In this more realistic case, due to the high dispersion of the path loss values with respect to the fitting model, Least Square and Non Linear Least Square methods have been implemented for the estimation of the in-body antenna coordinates. For both, 2D and $3 \mathrm{D}$, cases performance obtained under the assumption of ideal and not ideal channel estimation have been analyzed.

Results presented in this paper constitute a first step in the testing of RF-based localization techniques in more realistic environments compared to software simulations platforms.

\section{REFERENCES}

[1] G. Ciuti, A. Menciassi, and P. Dario, "Capsule Endoscopy: From Current Achievments to Open Challenges," IEEE Trans. Biomed. Eng., vol. 4, pp. 59-72, 2011.

[2] M. F. Hale, R. Sidhu, and M. E. McAlindon, "Capsule endoscopy: Current practice and future directions," World J. Gastroenterol., vol. 20, no. 24, pp. 7752-7759, 2014

[3] http://www.givenimaging.com/en-int/Innovative-Solutions/ProductSupport/pillcam-help-center/SoftwareInstructionalVideos/Pages.

[4] D. M. Pham and S. M. Aziz, "A real-time localization system for an endoscopic capsule using magnetic sensors," Sensors (Switzerland), vol. 14, no. 11, pp. 20910-20929, 2014.

[5] K. Pahlavan et al., "RF localization for wireless video capsule endoscopy," Int. J. Wirel. Inf. Networks, vol. 19, no. 4, pp. 326-340, 2012.

[6] M. Pourhomayoun, M. Fowler, and Z. Jin, "A novel method for medical implant in-body localization," in Proceedings of the Annual International Conference of the IEEE Engineering in Medicine and Biology Society, EMBS, 2012, pp. 5757-5760.

[7] C. Hu, M. Q. Meng, and M. Mandal, "Efficient Magnetic Localization and Orientation Technique for Capsule Endoscopy," in IEEE/RSJ International Conference on Intelligent Robots and Systems, 2005, pp. $3365-3370$.

[8] R. Kuth, J. Reinschke, and R. Rockelein, "Method for determining the position and orientation of an endoscopy capsule guided through an examination object by using a navigating magnetic field generated by means of a navigation device," US20070038063, 2007.

[9] G. Bao, L. Mi, and K. Pahlavan, "A Video Aided RF Localization Technique for the Wireless Capsule Endoscope (WCE) inside Small Intestine," in Proceedings of the 8th International Conference on Body Area Networks, 2013, no. February 2016, pp. 55-61.

[10] J. Lee, J. Oh, S. K. Shah, X. Yuan, and S. J. Tang, "Automatic classification of digestive organs in wireless capsule endoscopy videos," in Proceedings of the 2007 ACM symposium on Applied computing SAC 07, 2007, pp. 1041-1045.

[11] G. Bao and K. Pahlavai, "Motion estimation of the endoscopy capsule using region-based Kernel SVM classifier," in IEEE International Conference on Electro Information Technology, 2013.

[12] U. I. Khan, K. Pahlavan, and S. Makarov, "Comparison of TOA and RSS based techniques for RF localization inside human tissue," in Proceedings of the Annual International Conference of the IEEE Engineering in Medicine and Biology Society, EMBS, 2011, pp. 56025607.

[13] A. R. Nafchi, S. T. Goh, and S. A. R. Zekavat, "Circular arrays and inertial measurement unit for DOA/TOA/TDOA-based endoscopy capsule localization: Performance and complexity investigation," IEEE Sens. J., vol. 14, no. 11, pp. 3791-3799, 2014.

[14] Y. Geng and K. Pahlavan, "On the accuracy of RF and image processing based hybrid localization for wireless capsule endoscopy," in 2015 IEEE Wireless Communications and Networking Conference (WCNC), 2015, no. Wenc, pp. 452-457.

[15] G. Bao, "On simultaneous localization and mapping inside the human body (body-slam)," Ph.D. dissertation, ECE, WPI, Worcester, MA, USA, 2014.

[16] A. S. Bjørnevik, "Localization and Tracking of Intestinal Paths for
Wireless Capsule Endoscopy," M. S. Thesis, DET, Norwegian University of Science and Technology, 2015.

[17] M. R. Yuce, H. C. Keong, and M. S. Chae, "Wideband communication for implantable and wearable systems," IEEE Trans. Microw. Theory Tech., vol. 57, no. 10, pp. 2597-2604, 2009.

[18] Q. W. Jianqing Wang, Body Area Communications: Channel Modeling, Communication Systems, and EMC. 2013.

[19] H. Mateen, R. Basar, A. U. Ahmed, S. Member, and M. Y. Ahmad, "Localization of Wireless Capsule Endoscope : A Systematic Review," IEEE Sens. J., vol. 17, no. 5, pp. 1197-1206, 2017.

[20] B. Moussakhani, J. T. Flåm, S. Støa, I. Balasingham, and T. Ramstad, "On localisation accuracy inside the human abdomen region," IET Wirel. Sens. Syst., vol. 2, no. 1, pp. 9-15, Mar. 2012.

[21] M. Kanaan and M. Suveren, "In-body ranging with ultra-wideband signals: Techniques and modeling of the ranging error," Wirel. Commun. Mob. Comput., vol. 2017, 2017.

[22] Y. Ye, "Bounds on Rf Cooperative Localization for Video Capsule Endoscopy," Ph.D. dissertation, ECE, WPI, Worcester, MA, USA, 2013.

[23] S. Castello-Palacios, C. Garcia-Pardo, A. Fornes-Leal, N. Cardona, and A. Valles-Lluch, "Tailor-Made Tissue Phantoms Based on Acetonitrile Solutions for Microwave Applications up to $18 \mathrm{GHz}$," IEEE Trans. Microw. Theory Tech., vol. PP, no. 99, pp. 3987-3994, Nov. 2016.

[24] N. Cardona, S. Castello-Palacios, A. Fornes-Leal, C. Garcia-Pardo, and A. Valles-Lluch, "Synthetic Model of Biological Tissues for Evaluating the Wireless Transmission of Electromagnetic Waves," Patent WO/2017/109252, 30-Jun-2017.

[25] S. Castelló-palacios, C. Garcia-pardo, A. Fornes-leal, N. Cardona, and A. Vallés-lluch, "Full-Spectrum Phantoms for $\mathrm{cm}$-Wave and Medical Wireless Communications," pp. 1-3.

[26] S. Perez-Simbor, M. Barbi, C. Garcia-Pardo, "Initial UWB in-body channel characterization using a novel multilayer phantom measurement setup," in IEEE Wireless Communications and Networking Conference, WCNC, 2018.

[27] G. Imaging, "User Manual," no. November, 2016.

[28] C. Andreu, C. Garcia-Pardo, A. Fornes-Leal, M. Cabedo-Fabrés, and N. Cardona, "UWB In-Body Channel Performance by Using a Direct Antenna Designing Procedure," in 11th European Conference on Antennas and Propagation (EUCAP), 2017, p. 5.

[29] C. Tarín, P. Martí, L. Traver, N. Cardona, J. A. Díaz, and E. Antonino, "UWB channel measurements for hand-portable devices: A comparative study," in IEEE International Symposium on Personal, Indoor and Mobile Radio Communications, PIMRC, 2007.

[30] S. Perez-Simbor, C. Garcia-Pardo, C. Andreu, M. Frasson, N. Cardona, "Path Loss Models for Implanted Devices in the Gastrointestinal Area in the UWB Frequency Band," IEEE Trans. Antennas Propag., Special Issue on Wireless Healthcare Biotechnology, 2018, p. accepted.

[31] K. Arshak and F. Adepoju, "Adaptive linearized methods for tracking a moving telemetry capsule," IEEE Int. Symp. Ind. Electron., pp. 27032708, 2007.

[32] M. Barbi, S. Perez-Simbor, C. Garcia-Pardo, C. Andreu, N. Cardona, "Localization for Capsule Endoscopy at UWB Frequencies using an Experimental Multilayer Phantom," in IEEE Wireless Communications and Networking Conference, WCNC, 2018.

[33] W. Murphy and W. Hereman, "Determination of a position in three dimensions using trilateration and approximate distances," Department of Mathematical and Computer Sciences, Colorado School of Mines, Golden, Colorado, MCS-95, vol. 7. p. 19, 1995.

[34] Charles L. Lawson and Richard J. Hanson, Solving Least Squares Problems. 1995.

[35] I. Umay, B. Fidan, and B. Barshan, "Localization and tracking of implantable biomedical sensors," Sensors (Switzerland), vol. 17, no. 3, pp. 1-20, 2017.

[36] T. D. Than, G. Alici, H. Zhou and W. Li, "A Review of Localization Systems for Robotic Endoscopic Capsules," IEEE Trans. Biomed. Eng., vol. 59, no. 9, 2012.

[37] M. R. Basar, F. Malek, K. M. Juni, M. S. Idris, and M. I. M. Saleh, "Ingestible wireless capsule technology: A review of development and future indication," Int. J. Antennas Propag., vol. 2012, 2012.

[38] K. Krhac, K. Sayrafian, M. Alasti, K. Y. Yazdandoost, and D. Simunic, "A Study of Capsule Endoscopy Orientation Estimation Using Received Signal Strength,” PIMRC, 2018, pp. 345-349. 


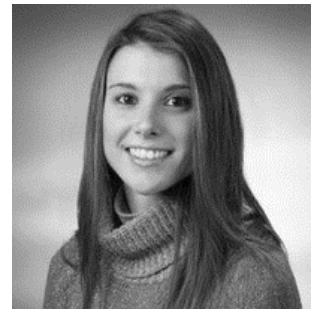

MARTINA BARBI was born in Reggio Emilia, Italy, in 1985. She received the M.S degree in telecommunication engineering from the University of Bologna, Italy, in 2012. From 2012-2016 she worked at the National Institute of Standards and Technology (NIST), in Gaithersburg, Maryland (USA) as contract researcher in the development of interference mitigation techniques for Body Area Networks (BANs).

She is currently a contract researcher within the WiBEC ITN (Wireless In Body Environment - Marie Curie Skłodowska Actions) at the Institute of Telecommunications and Multimedia Applications (iTEAM), UPV, Valencia, where she is pursuing her $\mathrm{PhD}$ degree in technologies for health and wellbeing.

Since 2016, she has been a researcher at iTEAM, Valencia. Her research interests include wireless medical communications, RF-based localization and tracking for capsule endoscopy at UWB frequencies. She is author or coauthor of 3 journal and 5 conference papers. Her current work is focused on the development and testing of localization techniques for capsule endoscopy applications at UWB frequencies.

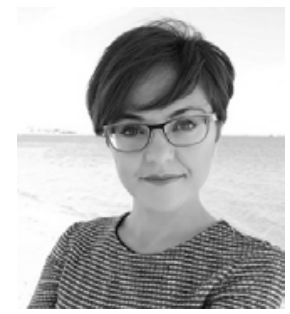

CONCEPCION GARCIA-PARDO attended the Universidad Politécnica de Cartagena, where she graduated in Telecommunication Engineering in 2007 and she received the M.Sc. degree in Information Technologies and Communications in 2008. In 2012, she received her $\mathrm{PhD}$ degree with European mention and qualification cum laude, from the Universidad Politécnica de Cartagena, and $\mathrm{PhD}$ degree in Microwaves and Microtechnologies with qualification Très Honorable from the Lille 1 University. Her $\mathrm{PhD}$ Thesis was awarded the special prize from the Universidad Politécnica de Cartagena in 2013.

In 2012, she joined the Institute of Telecommunications and Multimedia Applications (iTEAM) of the Universitat Politècnica de Valencia, Spain, where she works as senior researcher. She is author of more 40 publications of journal and conference papers related to wireless communications.

Dr. Garcia-Pardo has also participated in several national and international project related to wireless communications and wireless medical devices. She is also part of the management committee of COST Action CA 15104IRACON. She regularly serves as reviewer for the main journals related to electromagnetism and propagation. Her current work is focused on wireless medical devices and wireless communications for body area networks.

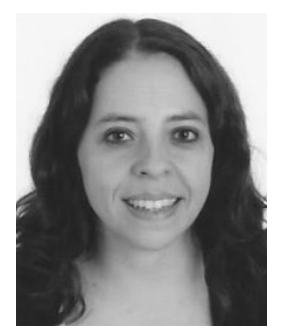

ANDREA NEVÁREZ was born in Quito, Ecuador. She received a medical doctor degree from Pontificia Universidad Católica of Ecuador in 2003. She completed her fellowship in Gastroenterology in University and Polytechnic Hospital La Fe in Valencia, Spain in 2010. She is currently pursuing her Ph.D. degree in the medical department at Universidad de Valencia in Spain.

In 2010 she attended for two months as an Exchange Fellow in MD Anderson Cancer Center in Houston, TX, USA. From $2010-2016$ has worked as a digestive endoscopist in her home country. She is currently working as a Ph.D. student at the University and Polytechnic Hospital La Fe in Valencia as an Early Stage Researcher for WiBEC ITN (Wireless In Body Environment - Marie Curie Skłodowska Actions). Her research interest include the development of medical devices and computer aided diagnosis systems applied to the digestive system.

MD Nevárez belongs to the Spanish Digestive Endoscopic Society (SEED) and is part of the study group in capsule endoscopy and enteroscopy. She has co-authored 4 papers published in indexed journals and also has presented oral communications in international meetings.



VICENTE PONS BELTRÁN was born in Alboraya, Valencia, Spain in 1965. Obtained his Gastroenterology fellowship in 1994 and his Ph.D. as a medical doctor from the University Miguel Hernández in 2001.

$\mathrm{He}$ is Head of the Digestive Endoscopy Unit at the University and Polytechnic Hospital La Fe in Valencia. Almost from the beginning of his professional career, he has developed his work linked to gastrointestinal endoscopy, primarily in the area of the biliopancreatic tree and small bowel endoscopy. He has conducted research projects on the field of endoscopic capsule and on the development of an enteroscopy prototype. He is the author of several international publications related to capsule endoscopy.

Dr. Pons Beltrán worked for more than 10 years together with researchers from the Polytechnic University of Valencia (Energy Engineering Institute, Department of Applied Physics, Technical college engineering design) and has led several research projects in the area of biomedical research (Endoworm project). He is regularly invited as a speaker at symposiums and conferences in the area of gastrointestinal endoscopy. At the time he is President of the Foundation of the National Spanish Digestive Endoscopy Society.

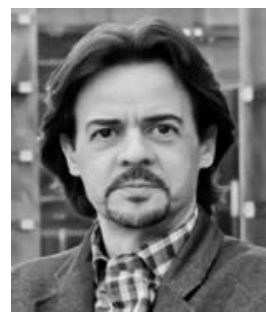

NARCíS CARDONA (M'91) was born in Barcelona, Spain. He received the M.S. degree in communications engineering from ETSI Telecommunications, Universitat Politècnica de Catalunya, Barcelona, in 1990, and the Ph.D. degree in telecommunications from the Universitat Politècnica de València, Valencia, Spain, in 1995

Since 1990, he is with the Communications Department of the Universitat Politècnica de València, and became Full Professor in 2006. He is the Head of the Mobile Communications Group and the Director of the Research Institute of Telecommunications and Multimedia Applications (iTEAM) of the Universitat Politècnica de València, a research institute with around 150 researchers including assistant professors \& research fellows. His current research interests include mobile channel characterization, broadcast cellular hybrid networks, and body area networks.

Prof. Cardona has been Vice-chairman of COST273, Chair of the WG3 in COST2100, General Chair of COST IC1004 and Vice-Chairman of COST IC15104 IRACON since 2016. He is also member of the Steering Committee of METIS (7FP), and METIS-II (H2020 5GPPP), WIBEC (H2020 ITN; 20162019), and coordinator of WaveComBE (ITN; 2017-2021). He has also organized several international conferences such as ISWCS 2006, IEEE PIMRC 2016, and EuCNC 2019. He is also author of 10 patents, several research books and more than 200 publications in the most relevant international journal and conferences of the communications field. 